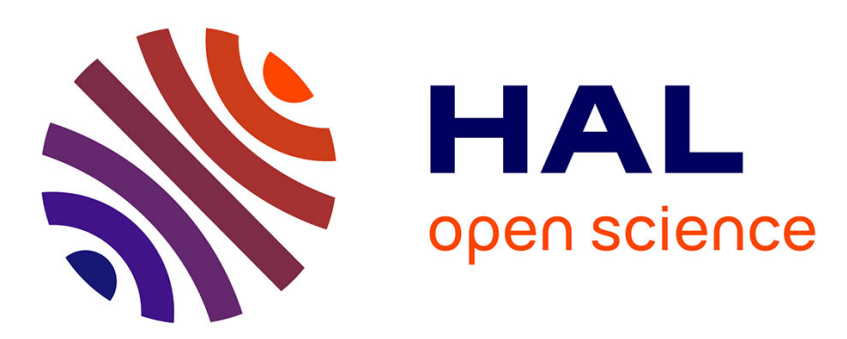

\title{
Multi-scale concrete model with rate-dependent internal friction
}

\author{
Ivan Giorgio, Daria Scerrato
}

\section{To cite this version:}

Ivan Giorgio, Daria Scerrato. Multi-scale concrete model with rate-dependent internal friction. European Journal of Environmental and Civil Engineering, 2016, 10.1080/19648189.2016.1144539 . hal01276774

\section{HAL Id: hal-01276774 \\ https://hal.science/hal-01276774}

Submitted on 20 Feb 2016

HAL is a multi-disciplinary open access archive for the deposit and dissemination of scientific research documents, whether they are published or not. The documents may come from teaching and research institutions in France or abroad, or from public or private research centers.
L'archive ouverte pluridisciplinaire HAL, est destinée au dépôt et à la diffusion de documents scientifiques de niveau recherche, publiés ou non, émanant des établissements d'enseignement et de recherche français ou étrangers, des laboratoires publics ou privés. 


\title{
Multi-scale concrete model with rate-dependent internal friction
}

\author{
Ivan Giorgio, Daria Scerrato
}

February 20, 2016

\author{
I. Giorgio (Corresponding author) - Dept. of Structural and Geotechnical Engineering, Università di \\ Roma La Sapienza, 18 Via Eudossiana, Rome, Italy \\ E-mail address of corresponding author: ivan.giorgio@uniroma1.it \\ I. Giorgio, D. Scerrato - International Research Center for the Mathematics and Mechanics of \\ Complex Systems MeMoCS, Università dell'Aquila, Cisterna di Latina, Italy
}

\begin{abstract}
In this paper, a micromorphic, non-linear 3D model aiming to describe internal friction phenomena in concrete is considered. A reduced two-degrees-of-freedom model is employed for the sake of easy handling to explain dissipative loops which have been observed in some concrete specimens tested under cyclic uniaxial compression loading with different frequencies and having various amplitudes but never inducing large strains. As (linear or non-linear) viscoelastic models do not seem suitable to describe neither qualitatively nor quantitatively the measured dissipation loops, we propose to introduce a multi-scale micro-mechanism of Coulomb-type internal dissipation associated to the relative motion of the faces of the micro-cracks present in the material and to the asperities inside the micro-cracks. We finally present numerical simulations showing that the proposed model is suitable to describe some of the available experimental evidence.

Keywords: Internal Friction, Nonlinear systems, LuGre model, dissipation in concrete, micromorphic continuum
\end{abstract}

\section{Introduction}

Concrete is the most widely employed structural material in the world, thus, its environmental impact is enormous considering the huge amounts of water and heat energy required to produce it, or the problem of its recycling when it is no longer usable. In this context, all efforts provided to improve concrete performance or its durability are really effective both from a social and an economic point of view. The paper is particularly focused on the design and characterization of earthquake-proof concrete, aiming to obtain a material with high durability and good performances for earthquake engineering. Indeed, some experimental campaigns [49] have shown that the addition of some ad-hoc fillers can significantly increase the internal dissipation under dynamic cyclic loading, even inside the conventional yield surface characterizing — with the commonly accepted approximation - the 
elastic domain. This first experimental evidence has justified the pursuit of the experiments for a larger range of frequencies [71] and the development of a novel constitutive model. Thus we have proposed such a model $[73,72]$ to take into account the internal friction phenomena occurring in the enriched concrete under cyclic loading and to provide a mathematical tool aiding in the design of advanced concretes with high dynamic performances for earthquake engineering.

Concrete is a highly heterogeneous material and has a complex microstructure. The microscopic complexity of such a material thus could be modeled, at a macro-level scale by higher gradient or micromorphic continua in the more general context of generalized materials (see for more details e.g. $[45,11,33,29])$; indeed, the standard Cauchy model is too poor to describe this material in our opinion. As a matter of fact concrete consists of a heterogeneous distribution of different kinds and amounts of solid phases, pores, and micro-cracks. By making suitable changes in the microstructure of this material, its properties can be modified (see e.g. [55]). Adding admixtures to the cement paste, it is possible to modify mechanical characteristics of the medium therefore pinpointing all the features of admixtures that are capable of improving the concrete dynamic behavior. Due to its multi-scale structure, this material can be susceptible to cracking when subjected to the influence of tensile stresses induced by differential movements between aggregates and cement paste. Such differential movements commonly arise, for example, either on drying or on cooling of concrete. A concrete may thus have micro-cracks even before it is loaded. Of course, impact loads, drying shrinkage, freeze and thaw cycles and sustained loads at high stress levels will have the effect of increasing the size and the number of micro-cracks. Micro-crack size can vary significantly, it may range from 0.1 to $500 \mu \mathrm{m}$ [54]. On the other hand, different concrete models are based on the idea of the existence of an interfacial transitional zone around aggregate grains, constituting a third phase, different from the aggregate and bulk cement paste phases (see e.g. [30, 83, 31] to model some relevant aspects of the interaction between different phases).

Many formulations have been proposed to account for the dissipation in solid media (see e.g. [12, $10,2,47,8]$ ), however, herein, with aiming at analyzing the internal mechanisms of dissipation in concrete when no relevant damage phenomena occur at the macroscopic level, the internal dissipation is assumed to be related to the relative motion of micro-crack faces following the same spirit of several papers $[46,43,59,36,81,84,78]$. The basic idea was to introduce a new (scalar) variable characterizing, in an average way, these micro-sliding along the micro-crack faces with a proper constitutive relation describing the evolution of this variable and its coupling with the Coulomb friction force. The validation of this model was fully convincing for a certain range of frequencies $[72,71]$-above $2 \mathrm{~Hz}$-, but it appears to not be applicable for lower frequencies. The purpose of this paper is now to introduce a second scalar variable to characterize not only the relative sliding of the micro-crack faces, but also the micro-structure of the asperities inside the micro-cracks - as a second step. These asperities are described by taking into account their heights through a probability distribution. During cyclic loading, all these asperity heights will essentially play a different role in a sliding direction and in its reverse, allowing to generalize the first model.

The paper is basically divided in three parts. In the first one, a review of dynamic friction models is provided by concluding the one which will be considered to build the present model. In the second part, the model is presented and the differences with the first model are emphasized and discussed. 
Table 1: Measured dissipated energy per cycle.

\begin{tabular}{lcccc}
\hline Frequency $(\mathrm{Hz})$ & 0.8 & 0.5 & 0.3 & 0.1 \\
\hline \hline Dissipated Energy $(\mathrm{J} / \mathrm{c})$ & $0.53 \pm 0.06$ & $0.92 \pm 0.03$ & $1.22 \pm 0.08$ & $1.60 \pm 0.02$ \\
\hline
\end{tabular}

Eventually, in final last part, the comparison with experimental results allows the investigation of the enlarged domain of validity of this new model particularly with respect to the frequency range.

\subsection{Motivation}

Since frictional forces arise as a results of a relative velocity of porous surfaces in a concrete matrix, we performed experimental tests in order to account for this issue. Specifically, we considered cyclic tests with varying frequency of load ranging from $0.1 \mathrm{~Hz}$ to $0.8 \mathrm{~Hz}$, i.e. relative micro-velocity, and with a load included between 19.62 and $392.4 \mathrm{kN}$. Following the procedure described in [49, 71], the dissipated energy for different kinds of specimen is estimated. Table 1 summarizes the dissipated energy for the different frequencies tested. In particular, Tab. 1 shows a relative increase compared to the frequency $0.8 \mathrm{~Hz}$ of $75 \%, 133 \%$ and $206 \%$ for the frequencies $0.5,0.3$ and $0.1 \mathrm{~Hz}$, respectively.

This analysis thus shows that the dissipated energy increases with decreasing frequency (see Fig. 1). This means that the friction in tested concrete presents a sort of 'Stribeck effect', i.e. a friction phenomenon that gives rise at low velocity decreasing friction with increasing velocity [76, 44]. To take this phenomenon into account, it is possible to address this issue with models that include this effect: examples of such a formulation are the LuGre model proposed by Canudas de Wit [23] or the Leuven model proposed by Swevers et al. [77].

\subsection{Friction models}

Friction phenomena occurring in solids should be models which belong to two main groups: i) static friction models derived from constitutive relationships, chosen to fit a specific behavior, between the friction force and a proper macroscopic variable, ii) dynamic friction models in which the friction force depends also on internal hidden variables that evolve according to differential equations conceived to match experimental measures [57, 80]. For instance, Coulomb model, which assumes the friction force to be independent of sliding velocity, falls into the first group. Many other examples can be taken into account; among these it is worth noting the static model proposed in [52]. The constitutive relation for the friction force $F$ in term of the sliding velocity $\dot{x}$ is

$$
F(\dot{x})=\gamma_{1}\left[\tanh \left(\gamma_{2} \dot{x}\right)-\tanh \left(\gamma_{3} \dot{x}\right)\right]+\gamma_{4} \tanh \left(\gamma_{5} \dot{x}\right)+\gamma_{6} \dot{x}
$$

where the term $\left[\tanh \left(\gamma_{2} \dot{x}\right)-\tanh \left(\gamma_{3} \dot{x}\right)\right]$ is related to the so-called Stribeck effect $[76,44]$, i.e. a phenomenon in which the increase of velocity implies a decrease of friction force; the second term 

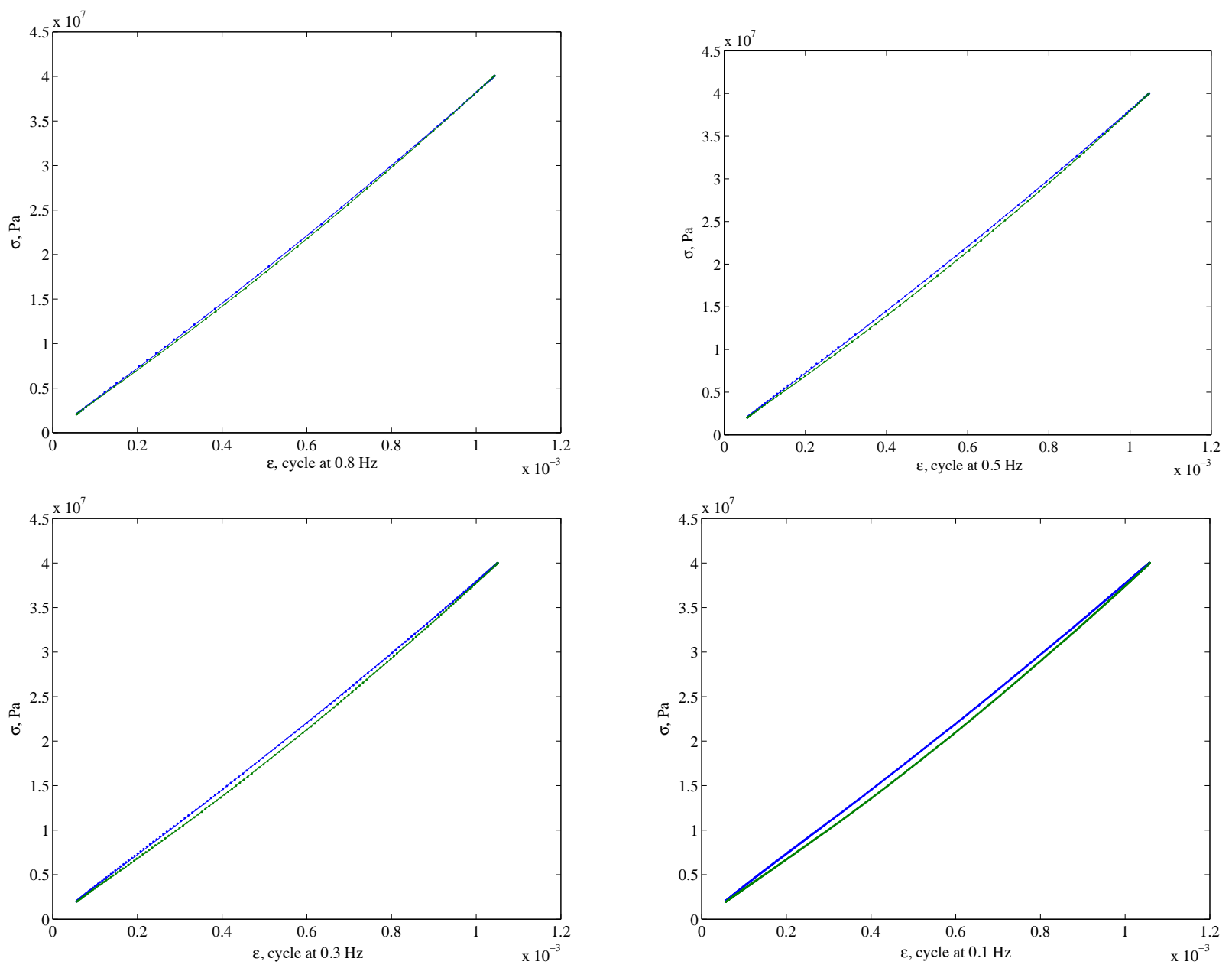

Figure 1: Measured dissipative loops for a concrete mixture and frequencies $0.8 \mathrm{~Hz}, 0.5 \mathrm{~Hz}, 0.3 \mathrm{~Hz}$ and $0.1 \mathrm{~Hz}$. 
is a regularization of the function signum characterizing the Coulomb behavior and the last term stands for a viscous effect that becomes significant at high velocities.

Several dynamic friction models have been proposed in literature. Among these, Dahl [20, 21] proposed a mathematical model of friction that was based on the assumption that the friction force $F$ is only a function of sliding displacement $x$, and monotonically approaches the Coulomb friction force $F_{C}$ as long as $\dot{x}$ is positive. When the direction of motion is reversed, the friction force approaches $-F_{C}$. The friction function slope $d F(x) / d x$, however, remains always positive, although $\dot{x}$ changes sign. This hysteretic behavior could be mimicked with a friction slope function of the form

$$
\frac{\mathrm{d} F(x)}{\mathrm{d} x}=\sigma\left|1-\frac{F}{F_{C}} \operatorname{sgn}(\dot{x})\right|^{\beta} \operatorname{sgn}\left(1-\frac{F}{F_{C}} \operatorname{sgn}(\dot{x})\right)
$$

where the quantity $\sigma$ is the slope of the force-deflection curve at $F=0$ or the rest stiffness. The value of the parameter $\beta$ defines the basic shape of the friction function and thus it is chosen to characterize the friction law being used. A more useful way to employ Dahl's assumption consists in considering the time evolution of the friction force by means of the simple equation $\dot{F}=F^{\prime} \dot{x}$. The Dahl friction force could be interpreted as a nonlinear elastoplastic model with a nearly linear elastic behavior for small deflections, which approaches a plastic behavior for large deflections (see Fig. 2). Figure 2 shows typical Dahl's cycles obtained by varying the rest stiffness $\sigma$ in dimensionless form.

In [22] Dankowicz suggests that the origin of frictional force between two rough solid surfaces coming into contact lies in the interaction of their asperities. The asperities are assumed to have different heights — relative to a reference plane - according to a probability distribution, while all the other properties remains unchanged. The imposition of a tangential displacement entails that the shorter asperities, which are under less compression, begin to slide before the longer ones and, only after a sufficiently large tangential displacement, the resultant tangential load is within a given deviation from maximum tangential load. To take account of this quasi-static behavior, an average shearing deformation, $z$, is introduced which describes the collective asperity deformation and the friction force is assumed as

$$
F=F_{\operatorname{Max}} \frac{z}{\delta}=\mu N e^{-\Delta d / \mathrm{s}} \frac{z}{\delta}
$$

where $\mu$ is the friction coefficient, $N$ is the normal load applied, $\Delta d$ is the change in distance between the reference planes of the interacting surfaces, $\mathrm{s}$ is the standard deviation of the probability distribution of asperity heights and $\delta$ is the average deformation obtained under maximum tangential load. To consider the lag between the change in direction of motion and the tangential load, the deformation $z$ is described by an ordinary differential equation according to the evolution rule

$$
\frac{\mathrm{d} z}{\mathrm{~d} t}=\dot{x}\left(1-\operatorname{sgn}(\dot{x}) \frac{z}{\delta}\right)
$$

$x$ being the macroscopic tangential displacement.

The so-called Lund-Grenoble (LuGre) model proposed in [23] is also based on the average behavior of the bristles whose average deflection is denoted by $z$. Similarly to the previous model, the 
evolution equation is

$$
\frac{\mathrm{d} z}{\mathrm{~d} t}=v-\frac{|v|}{g(v)} z
$$

in which $g$, i.e. the maximum value of $z$, depends explicitly on the sliding velocity. In the Dankowicz model a dependence on the sliding velocity is introduced in the maximum shear force through the surface separation $\Delta d$ considered as a dynamic variable evolving according to its own differential equation. The expression assumed for $g$ to describe the Stribeck effect is

$$
\sigma_{0} g(v)=F_{C}+\left(F_{S}-F_{C}\right) \exp \left[-\left(\frac{v}{v_{s}}\right)^{2}\right]
$$

where $\sigma_{0}$ is the rest stiffness, $F_{C}$ is the Coulomb friction force, $F_{S}$ is the stiction force which plays the role of a static friction force, and $v_{s}$, is a reference velocity also called the Stribeck velocity. The constitutive relationship for the friction force due to the bending of the bristles is given by

$$
F=\sigma_{0} z+\sigma_{1} \dot{z}+\sigma_{2} v
$$

The Dahl model is based on a Coulomb friction with a lag in the change of the friction force when the direction of motion reverses. The LuGre model generalizes the model of Dahl enabling it to describe the Stribeck effect, in which at low-velocity range the friction force decreases as velocity increases. Figure 3 shows, in dimensionless form, the dependence of LuGre's model on the sliding velocity. We can observe that when the maximum velocity of the cycle approaches zero the friction force tends to the static friction force $F_{S}$, while for high velocities the friction force is about equal to the Coulomb friction $F_{C}$. Anyway, if $F_{C}=F_{S}$ there is not Stribeck effect and the LuGre model, which is rate dependent, reduces to the Dahl model, which is rate independent.

Finally it is worth mentioning the Leuven model, an extension of the LuGre model, proposed by Swevers et al. in [77]. The key idea is to fit transition curves of arbitrary forms. The friction force is modeled as function of an auxiliary variable $z$, namely the average deformation of the asperities of the contact surfaces. The constitutive equation for the friction force is

$$
F=F_{h}(z)+\sigma_{1} \dot{z}+\sigma_{2} v
$$

where $F_{h}(z)=F_{b}+F_{d}(z)$ is the hysteresis friction force. $F_{d}(z)$ is the transition curve which is active at a certain time, $F_{b}$ is the value of the friction force at the beginning of a transition curve. The evolution equation of the state variable $z$ is

$$
\frac{\mathrm{d} z}{\mathrm{~d} t}=v\left(1-\operatorname{sgn}\left(\frac{F_{d}(z)}{S(v)-F_{b}}\right)\left|\frac{F_{d}(z)}{S(v)-F_{b}}\right|^{\beta}\right)
$$

in which the function $S(v)$ characterizes the sliding regime near zero velocity, and is given by 


$$
S(v)=F_{C}+\left(F_{S}-F_{C}\right) \exp \left(-\left|\frac{v}{v_{s}}\right|^{\delta}\right)
$$

In order to achieve a better match between model predictions and the experimental tests performed and recalled in Sec. 1.1, we propose to exploit a LuGre-type model adapted to the continuous case to take account of a 'Stribeck effect', as will be explained in detail in the rest of the article.

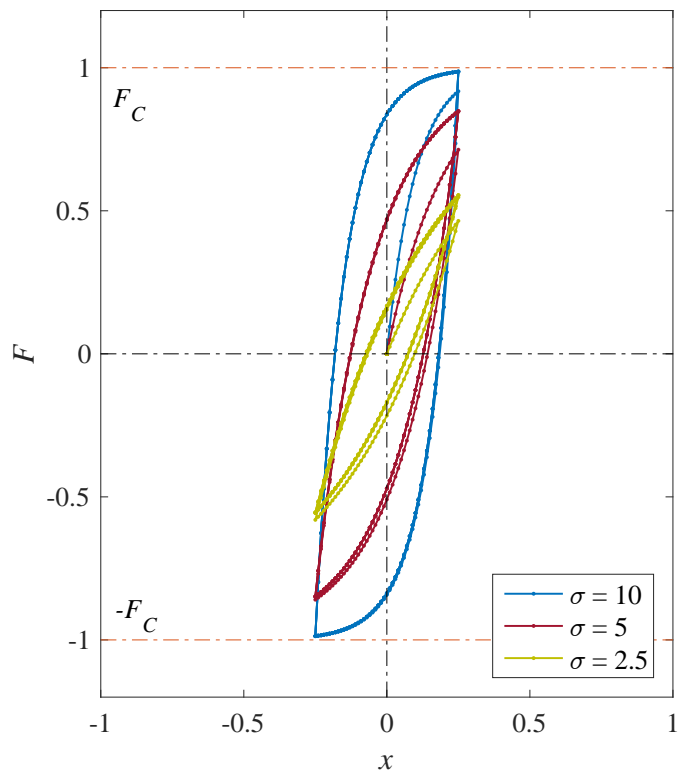

Figure 2: Tipical Dahl's cycles

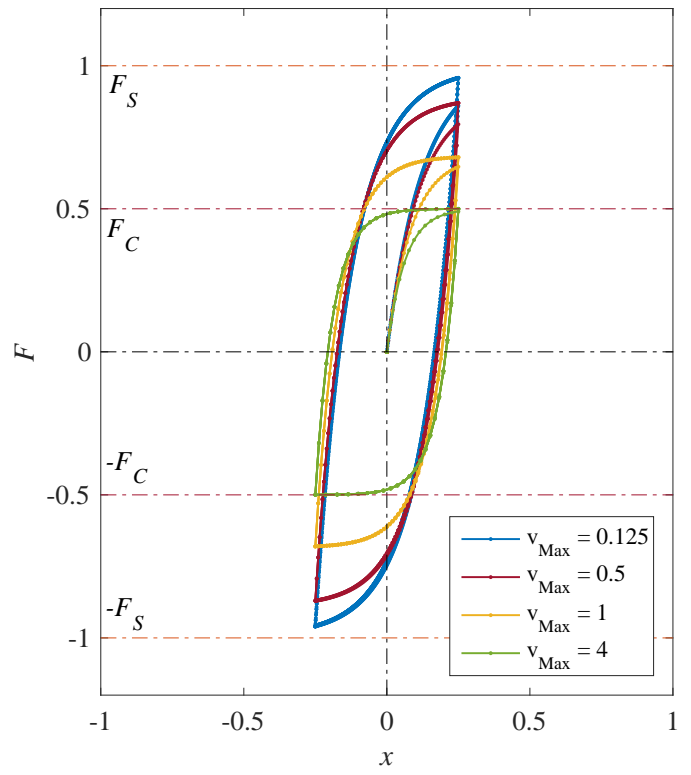

Figure 3: Tipical LuGre's cycles

\section{Modeling}

Multi-scale models are a powerful tool to deal with complex materials which are increasingly used in engineering applications. Specifically, motivated by the studies referred to above, a model is herein developed for an engineering material which is a standard or enriched concrete. This building material has to be investigated from a dynamical point of view with three different scales, a bulk scale, a meso-scale of pores inside the solid matrix and finally a micro-scale of asperities characterizing the porous surfaces that come into contact with each other under a compression (see Fig. 4). The results obtained employing the proposed model may depend on the shape of interfaces which can be treated as rough surfaces. For such type of interfaces the averaging procedure proposed in [37] using various models of surface elasticity can be taken into account. The model for the macro-scale is based on a 


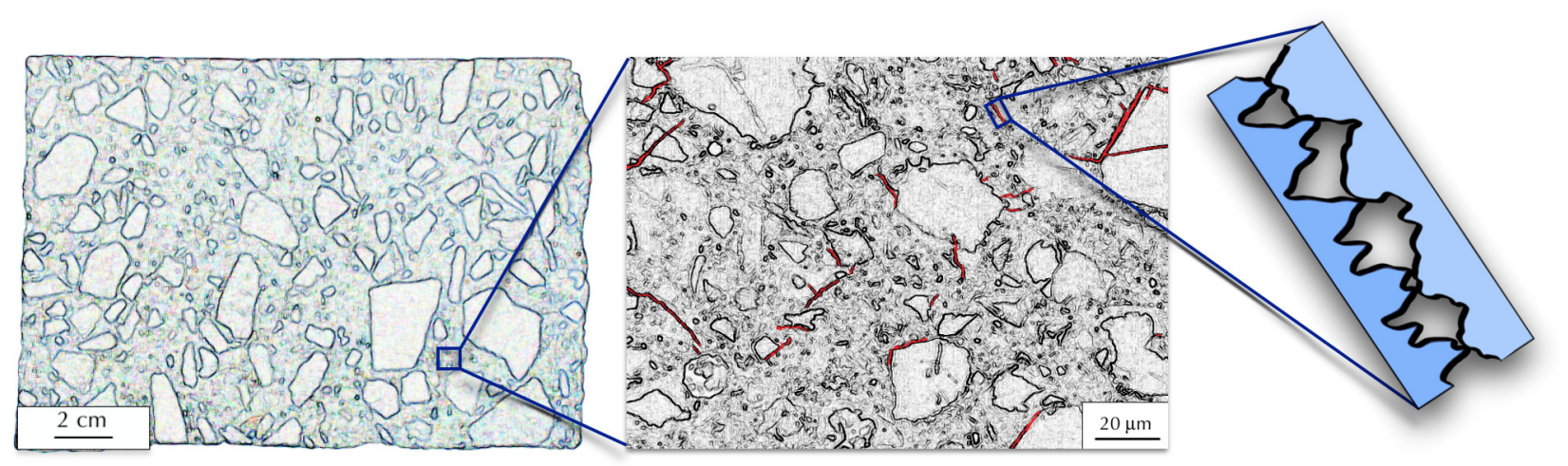

Figure 4: Three scale representation.

Saint-Venant theory (see for more details [73] and [26]). The constitutive equations at the meso-scale contains elastic nonlinearities, which can be observed through experimental testings. The model for the micro-scale is derived from the LuGre model that accounts for Coulomb friction and includes the Stribeck effect. The proposed model is encompassed in the framework of the micromorphic continuum theory (many examples of such an approach can be found in the literature, see e.g. [82, $6,51,53,67,65,28,66])$. Thus, to obtain a proper kinematical description aiming to describe internal friction phenomena, we choose the common variable $\boldsymbol{u}$ as a displacement field defined on $\mathscr{B}$ (a simple connected open domain), the meso-structural variable $\varphi$ as a scalar field, which may be interpreted as average slip inside the concrete matrix, i.e. an average relative displacement between opposite faces of microcracks, and the micro-structural variable $z$ as a scalar field which describes the collective asperity deformation.

In this framework, the 'internal forces' acting on a given subdomain $\mathscr{D}$ of $\mathscr{B}$ have a virtual work which may be written as

$$
\delta \mathscr{W}_{(\mathrm{i})}=-\int_{\mathscr{D}} \delta \Psi(\boldsymbol{u}, \varphi) \mathrm{d} V
$$

$\Psi$ being the elastic potential (see $[73,72]$ )

$$
\Psi=\frac{1}{2} \lambda[\operatorname{tr}(E)]^{2}+\mu \operatorname{tr}\left(E^{2}\right)+\frac{1}{2} k_{1} \varphi^{2}+\frac{1}{3} k_{2} \varphi^{3}+\frac{1}{4} k_{3} \varphi^{4}+\alpha \varphi \sqrt{\mathrm{II}_{\operatorname{dev} E}}
$$

where $E$ is the linearized strain tensor, $\mathrm{II}_{\mathrm{dev} E}{ }^{1}$, related to the coupling term, is the second invariant of the deviatoric component of $E$, and represents a measure of shear deformations that is responsible for a relative displacement $\varphi$. This representation of the coupling term is very close to the common expression used in porous materials (see e.g. [7, 18] and more recently [74, 32, 64]), even if in this

\footnotetext{
${ }^{1} \mathrm{II}_{\operatorname{dev} E}=\frac{1}{2} \operatorname{tr}\left[(\operatorname{dev} E)^{2}\right]$, where the deviatoric strain tensor $\operatorname{dev} E=E-\frac{1}{3} \operatorname{tr}(E) \mathbb{1}$.
} 
case, we use $\mathrm{II}_{\mathrm{dev} E}$ instead of the trace of the small strain tensor; this choice is dictated by our physical interpretation of $\varphi$; the exchange of energy between the bulk and the microstructure is related to a deformation at macro level that induces a sliding on micro-cracks. In Eq. (12) the microstructure elastic force is assumed to be nonlinear. It is well-known that for a low level of external static compression the behavior of the concrete can be assumed in a satisfactory way linear. Nevertheless, tests performed with cyclic loads show that even in a small strain range the stressstrain relationship appears to be nonlinear. Therefore, the presence of an internal phenomenon that triggers the nonlinear behavior in presence of varying loads can be assumed. It is reasonable to attribute this effect to the microstructure involved in the micro-sliding. In other words, the presence of micro-cracks, very reasonably, introduces non-linear effects that are activated by time-varying loads (see for similar effects e.g. [61, 62, 56]). We notice that the most convenient pair of elastic constants for the expression of the stored energy density of an isotropic material is that comprised of the bulk modulus $\kappa$ and the shear modulus $\mu[38,39,40]$, even more so than the pair of Lamé's constants.

The 'external forces' acting on $\mathscr{D}$ are: i) volume forces, exerted on $\mathscr{D}$ by systems located outside $\mathscr{B}$, giving the virtual work

$$
\delta \mathscr{W}_{(\mathrm{v})}=\int_{\mathscr{D}} f_{i} \delta u_{i} \mathrm{~d} V
$$

ii) contact forces provided on $\mathscr{D}$ by the parts of $\mathscr{B}$ located outside $\mathscr{D}$, giving the virtual work

$$
\delta \mathscr{W}_{(\mathrm{s})}=\int_{\partial \mathscr{D}} \tau_{i} \delta u_{i} \mathrm{~d} S
$$

where $\tau_{i}$ is the usual traction acting on the boundary $\partial \mathscr{D}$ (see e.g. $[60,15,48]$ ). The virtual work due to inertial forces can be expressed by

$$
\delta \mathscr{W}_{(\mathrm{in})}=-\int_{\mathscr{D}} \rho \ddot{u}_{i} \delta u_{i} \mathrm{~d} V-\int_{\mathscr{D}} \rho_{\varphi} \ddot{\varphi} \delta \varphi \mathrm{d} V
$$

where $\rho$ and $\rho_{\varphi}$ are the (apparent) mass density of bulk material and the effective mass density linked to the micro-structural variable $\varphi$ respectively. The virtual work of friction forces can be specified as

$$
\delta \mathscr{W}_{(\mathrm{d})}=-\int_{\mathscr{D}} F \delta \varphi \mathrm{d} V
$$

where, according to LuGre model, the tangential load can be assumed

$$
F=F_{\mathrm{Max}} \frac{z}{\delta\left(\sigma_{n}, \dot{\varphi}\right)}+\gamma_{1} \dot{z}+\gamma_{2} \dot{\varphi}
$$

being $\gamma_{1}$ and $\gamma_{2}$ damping coefficients related to viscous effects at high frequencies, $\delta$ the collective maximum deformation under the maximum tangential load, i.e. 


$$
F_{\text {Max }}=\mu(\dot{\varphi}) \varpi \mathfrak{h}\left(\sigma_{n}\right)
$$

which results in a Coulomb-like expression, where $\mu$ is the friction coefficient, $\varpi$ is the specific surface of micro-cracks, i.e. the surface area of micro-cracks per unit volume of concrete and $\mathfrak{h}$ is a suitable function of $\sigma_{n}=\kappa \operatorname{tr}(E)$ i.e. the compressive stress that allows the contact of the opposite faces of the micro-cracks. The function $\mathfrak{h}\left(\sigma_{n}\right)$ takes into account the 'real' condition of contact (Signorini-like conditions [75]), indeed it can be expressed as

$$
\mathfrak{h}\left(\sigma_{n}\right)= \begin{cases}0 & \left|\sigma_{n}\right|<\sigma_{T} \\ \sigma_{n} & \left|\sigma_{n}\right| \geqslant \sigma_{T}\end{cases}
$$

where $\sigma_{T}$ is a threshold stress denoting the minimum pressure required to close the microcracks and assure the contact between the opposite faces. Clearly, when $\left|\sigma_{n}\right|$ is less than $\sigma_{T}$ there is not contact at all and then friction force can not be exerted. In future developments, we can improve this contact model by involving also the presence of a micro-impact between the opposite faces of microcracks (see on this subject e.g. [3, 4]). In particular, the friction force can be rearranged $F_{\mathrm{Max}}=$ $\tilde{\mu}(\dot{\varphi}) \zeta \hat{\mathfrak{h}}[\operatorname{tr}(E)]^{2}$ introducing only one material parameter $\zeta$ defined as $\mu_{s} \varpi \kappa$ and the dimensionless coefficient $\tilde{\mu}$ chosen to yield a steady-state dependence of friction on the slide velocity $\dot{\varphi}$ which agrees with a Stribeck curve, as follows ${ }^{3}$

$$
\tilde{\mu}=\frac{\mu(\dot{\varphi})}{\mu_{s}}=\frac{\mu_{k}}{\mu_{s}}+\left(1-\frac{\mu_{k}}{\mu_{s}}\right) \exp \left(-\left|\frac{\dot{\varphi}}{v_{s}}\right|^{\varsigma}\right)
$$

Parameters $\mu_{s}, \mu_{k}$ and $v_{s}$ are respectively the static friction, the kinetic friction and the Stribeck velocity which must be matched to experimental observations. The exponent $\varsigma$ is a parameter which can be chosen in the range from 0.5 to 2 (see [9]). The collective maximum deformation $\delta$ is assumed to be

$$
\delta\left(\sigma_{n}, \dot{\varphi}\right)=\frac{F_{\mathrm{Max}}}{\gamma_{0}}
$$

By combining the expressions above we assume the following expression for the total tangential component of the surface interactions, or the friction force

$$
F=\gamma_{0} z+\gamma_{1} \dot{z}+\gamma_{2} \dot{\varphi}
$$

where $\gamma_{0}$ is the stiffness. As a result, according to the point of view of Dahl and LuGre models, an evolution equation for the variable $z$ can be assumed as

\footnotetext{
${ }^{2}$ Where the function $\hat{\mathfrak{h}}$ is defined from the Eq. (19), by considering the relation: $\sigma_{n}=\kappa \operatorname{tr}(E)$.

${ }^{3}$ In a similar way a Lorentzian function can be used: $\tilde{\mu}=\frac{\mu(\dot{\varphi})}{\mu_{s}}=\frac{\mu_{k}}{\mu_{s}}+\left(1-\frac{\mu_{k}}{\mu_{s}}\right) \frac{1}{1+\left|\frac{\dot{\varphi}}{v_{s}}\right|^{\varsigma}}$.
} 


$$
\dot{z}=\dot{\varphi}\left|1-\frac{z}{\delta(\operatorname{tr}(E), \dot{\varphi})} \operatorname{sgn}(\dot{\varphi})\right|^{\beta} \operatorname{sgn}\left(1-\frac{z}{\delta(\operatorname{tr}(E), \dot{\varphi})} \operatorname{sgn}(\dot{\varphi})\right)
$$

The generalized principle of virtual work then becomes

$$
\delta \mathscr{W}_{(\mathrm{i})}+\delta \mathscr{W}_{(\mathrm{v})}+\delta \mathscr{W}_{(\mathrm{s})}+\delta \mathscr{W}_{(\mathrm{in})}+\delta \mathscr{W}_{(d)}=0
$$

The proposed model can be fruitfully applied to the general case of triaxial compression loads. Indeed, concrete is commonly subjected to triaxial stress states, and it is well known that the stress level influences greatly its mechanical behaviour. The Eq. (24) can be solved with the Finite Element Method; however, given the complexity of the material under study, some computation issues such as locking or hourglass modes can arise. To tackle these problems an isogeometric analysis can be employed as a powerful numerical tool (on this formulation some relevant works are e.g. $[19,41,79,14,13,25])$. On the other hand, based-cement materials can exhibit an internal loss of stability due to the presence of high heterogeneous phases inside their solid matrix. To investigate this disagreeable behavior, we refer to some relevant analyzes [68, 70, 69].

To analyze the case of a simple compression (with Poisson effect) in cylindrical concrete specimens the Eq. (24) can be reduced to a system of OD equations much easier to handle. In the framework of the Saint-Venant theory, we can assume as kinematical descriptor for the bulk deformation, the strain along the longitudinal axis of the considered cylinder, $\varepsilon$. In this case this strain field is a scalar variable that does not depend on spatial coordinates, but only, possibly on time. The same assumption of a spatial uniform field is made even for the variable $\varphi$ because of the assumed uniformly distributed random orientation of the micro-cracks and for the compressive load considered that is also uniform. With these assumption, the principle of virtual work (24) for any arbitrary virtual strain $\delta \varepsilon$ and virtual micro-sliding $\delta \varphi$ provides

$$
\left\{\begin{array}{l}
M_{\varepsilon} \ddot{\varepsilon}+K_{\varepsilon} \varepsilon+\tilde{\alpha} \varphi=f^{e x t}(t) \\
m_{\varphi} \ddot{\varphi}+k_{\varphi}(\varphi)+\tilde{\alpha} \varepsilon+F(z, \dot{z}, \dot{\varphi})=0 \\
\dot{z}=\dot{\varphi}\left|1-\frac{z}{\delta(\varepsilon, \dot{\varphi})} \operatorname{sgn}(\dot{\varphi})\right|^{\beta} \operatorname{sgn}\left(1-\frac{z}{\delta(\varepsilon, \dot{\varphi})} \operatorname{sgn}(\dot{\varphi})\right)
\end{array}\right.
$$

where the non-linear microstructure elastic force is expressed by the power-law below

$$
k_{\varphi}(\varphi)=\tilde{k}_{1} \varphi+\tilde{k}_{2} \varphi^{2}+\tilde{k}_{3} \varphi^{3}
$$

$\beta$ is a parameter that defines the force-deflection curve, and the material parameters can be deter- 
Table 2: Values of the material parameters used in numerical simulations.

\begin{tabular}{ccccc}
\hline$K_{\varepsilon}(\mathrm{Nm})$ & $\tilde{\alpha}(\mathrm{N})$ & $\tilde{k}_{1}(\mathrm{~N} / \mathrm{m})$ & $\tilde{k}_{2}\left(\mathrm{~N} / \mathrm{m}^{2}\right)$ & $\tilde{k}_{3}\left(\mathrm{~N} / \mathrm{m}^{3}\right)$ \\
\hline \hline $1.27 \times 10^{8}$ & $3.29 \times 10^{8}$ & $2.12 \times 10^{9}$ & $2.64 \times 10^{5}$ & $1.71 \times 10^{16}$ \\
\hline
\end{tabular}

mined by

$$
\begin{gathered}
M_{\varepsilon}=\int_{\mathscr{B}} \rho\left(\nu^{2}\left(x_{1}^{2}+x_{2}^{2}\right)+x_{3}^{2}\right) \mathrm{d} V, \quad m_{\varphi}=\int_{\mathscr{B}} \rho_{\varphi} \mathrm{d} V \\
K_{\varepsilon}=\int_{\mathscr{B}} \frac{\mu(3 \lambda+2 \mu)}{\lambda+\mu} \mathrm{d} V=\int_{\mathscr{B}} Y \mathrm{~d} V, \quad \tilde{\alpha}=\int_{\mathscr{B}}(\sqrt{3} / 3(1+\nu) \alpha) \mathrm{d} V \\
\tilde{k}_{1}=\int_{\mathscr{B}} k_{1} \mathrm{~d} V, \quad \tilde{k}_{2}=\int_{\mathscr{B}} k_{2} \mathrm{~d} V, \quad \tilde{k}_{3}=\int_{\mathscr{B}} k_{3} \mathrm{~d} V
\end{gathered}
$$

being $Y$ and $\nu$ respectively the Young modulus and the Poisson ratio. For the determination of all of these constitutive parameters, it is possible to resort to methods of identification as outlined in $[63,35,24]$.

Note that since $z$ is governed by an ordinary differential equation, it does not respond instantaneously to changes of slide velocity. This accounts for the lag between the change in direction of motion and the tangential load. It is worth noting that the LuGre model also satisfies a Lipschitz condition in $z$ if $\beta \geqslant 1$ and therefore has a unique solution; otherwise, if $0 \leqslant \beta<1$, the Lipschitz condition is not satisfied but only the continuity [58].

Friction dissipates energy, however, since this model is dynamic, there may be time intervals where frictional visco-plasticity stores energy and others where it gives energy back. Barabanov and Ortega provided an answer to the question of passivity of the generalized LuGre friction model. The concept of passivity in this context is related to a dissipative system in which any motion starting at the time $t_{0}$ satisfies for all time $T$ the inequality

$$
\wp=\int_{t_{0}}^{T} F \dot{\varphi} \mathrm{d} t \leqslant 0
$$

Namely, they have shown that the model defines a passive operator $\wp$ if and only if the coefficients satisfy a proper inequality [5].

\section{Results and discussion}

To illustrate the features of the proposed model, we consider a basic example using some concrete specimens undergoing cyclic uniaxial compression loading with different frequencies. In this case Eqs. (25) can be utilized once all the material parameters are estimated on the base of a cylindrical sample whose diameter is $D=11.28 \mathrm{~cm}$ and having height $h=22 \mathrm{~cm}$. Some criteria have been taken 
Table 3: Simulated dissipated energy per cycle.

\begin{tabular}{lcccc}
\hline Frequency $(\mathrm{Hz})$ & 0.8 & 0.5 & 0.3 & 0.1 \\
\hline \hline Dissipated Energy $(\mathrm{J} / \mathrm{c})$ & 0.59 & 0.93 & 1.31 & 1.61 \\
\hline
\end{tabular}

into account for parameter identification. Indeed, values of the parameter $\varphi$ have to be compatible with the order of magnitude of known dimensions of typical cracks in concrete. The micro-force $k_{\varphi}(\varphi)$ is assumed to be elastic, and so monotonously increasing. The deformation energy needs to be definite positive and consequently suitable restrictions on the stiffness parameters as well as on the coupling between micro- and macro-motion must be considered. Table 2 summarizes the material parameters used in the simulations. We remark that non-linear effects are not negligible as it is clear from the non-linear stiffnesses. The equivalent mass $M_{\varepsilon}$ can be easily estimated by assuming that the volume mass density of material is homogeneous - in an averaged sense - and known, here it has been set $\rho=2300 \mathrm{~kg} / \mathrm{m}^{3}$. The order of magnitude of the micro-parameter $m_{\varphi}$, not directly measurable, was selected in order to better fit with experimental data, assuming reasonably that the micro-mass density, $\rho_{\varphi}$ is a suitable fraction of the bulk mass density $\rho$, here we set $\rho_{\varphi}=\rho / 10$. The other parameters used in the simulations and related to the dissipation are $\mu_{k} / \mu_{s}=0.2, \varsigma=2, \beta=1, \gamma_{0}=5 \times 10^{11} \mathrm{~N} / \mathrm{m}^{4}, v_{s}=1.9 \times 10^{-4} \mathrm{~m} / \mathrm{s}, \gamma_{1}=1 \mathrm{~N} \mathrm{~s} / \mathrm{m}^{4}, \gamma_{2}=2 \times 10^{8}$ $\mathrm{N} \mathrm{s} / \mathrm{m}^{4}$.

The cyclic external load applied is $f^{e x t}(t)=-F_{0}-F_{1} \sin (2 \pi f t)$ with a pre-load $F_{0}$ to ensure that we simulate a regime in which the micro-cracks are closed, and with frequency $f=\{0.1,0.3,0.5,0.8\}$ $\mathrm{Hz}$. The values of $F_{0}$ and $F_{1}$ are respectively $206.01 \mathrm{kN}$ and $186.39 \mathrm{kN}$, in order to have the same setup of the experimental tests.

Figure 3 shows the dissipative loops in a stress-strain plane obtained varying frequency. In this range of frequencies it is easy to observe that the dissipation energy, i.e. the area of the loops, decreases with increasing of frequency. That particular behavior -indeed, it is opposite to the behavior expected for a viscous dissipation- matches with measured cycles shown in Fig. 1. In Tab. 3 are reported the dissipated energy for the frequencies tested as obtained by the numerical simulations. Comparing these values with those measured and listed in Tab. 1, we can observe a good matching between previsions of the proposed model and measurements.

Figure 6 displays the friction force for two cycles of frequency $0.1 \mathrm{~Hz}$. It is possible to note the sharp shape of the force in the neighborhood of reversal velocities points at $0,5,10$ and $15 \mathrm{~s}$. In addition, between these reversal points, at the center of the loading and unloading curves, it is possible to recognize the effect due to the pressure $\sigma_{n}$; indeed, the amplitude of the friction force is varied in accordance to the amplitude of the modulating pressure $\sigma_{n}$, which change the level of the interaction between the faces of the micro-cracks, implying a greater or lesser friction force.

Figure 7 exhibits the power $\wp$ of the friction force $F$. It is worth noting that even if some instantaneous values of the power are positive, the passivity of the system considered, which is recalled in Eq. (27), is respected. This behavior can be justified attributing, as predicted by the 

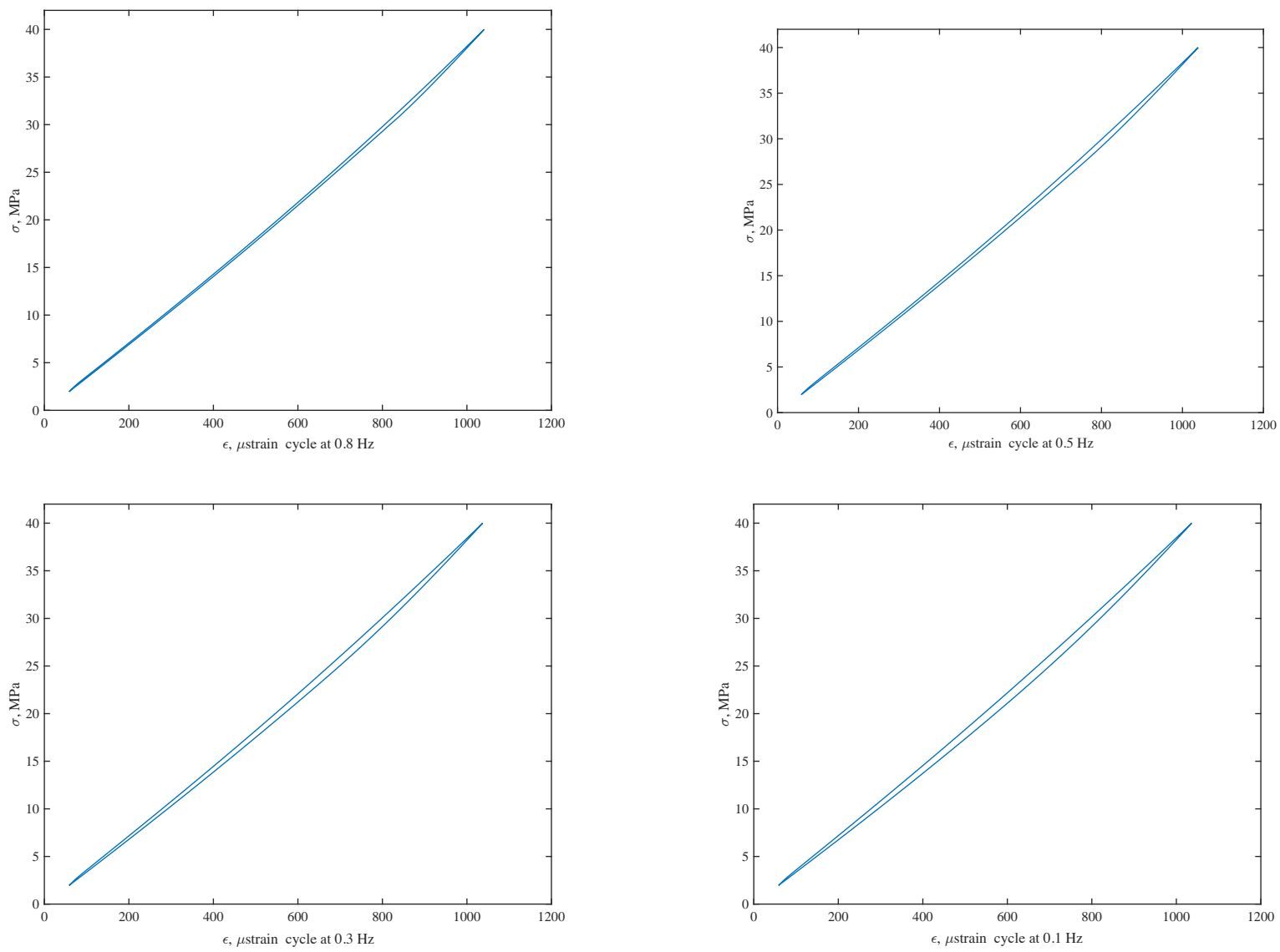

Figure 5: Simulated dissipative loops for a concrete mixture and frequencies $0.8 \mathrm{~Hz}, 0.5 \mathrm{~Hz}, 0.3 \mathrm{~Hz}$ and $0.1 \mathrm{~Hz}$. 


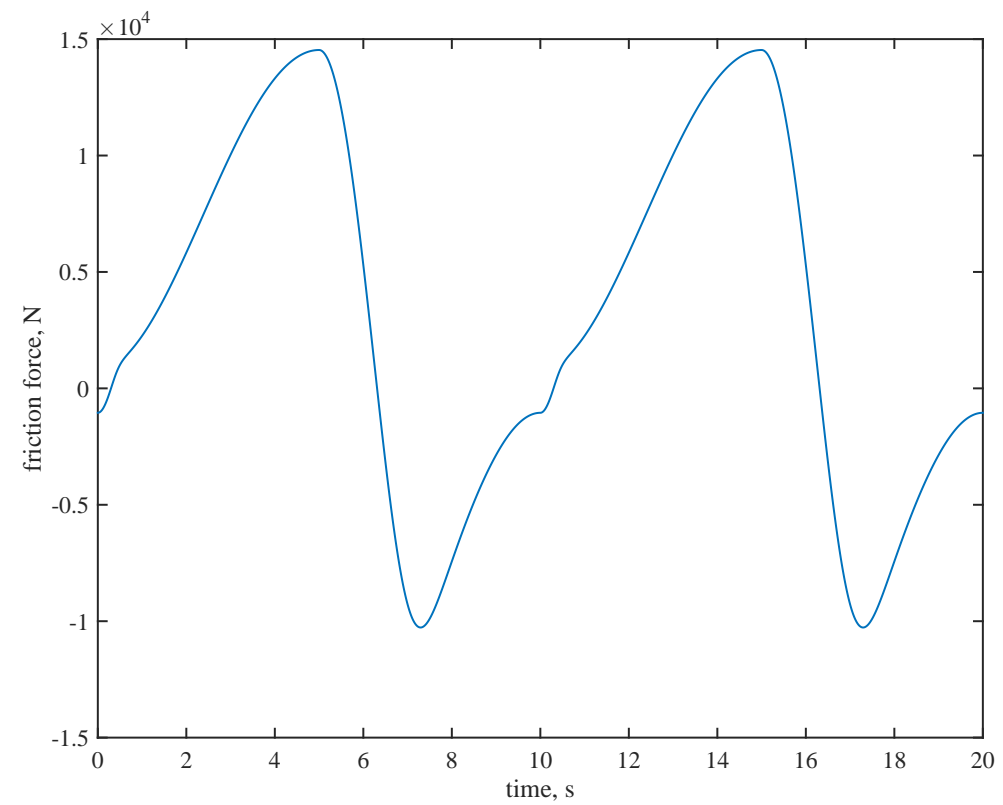

Figure 6: Friction force for two cycles 


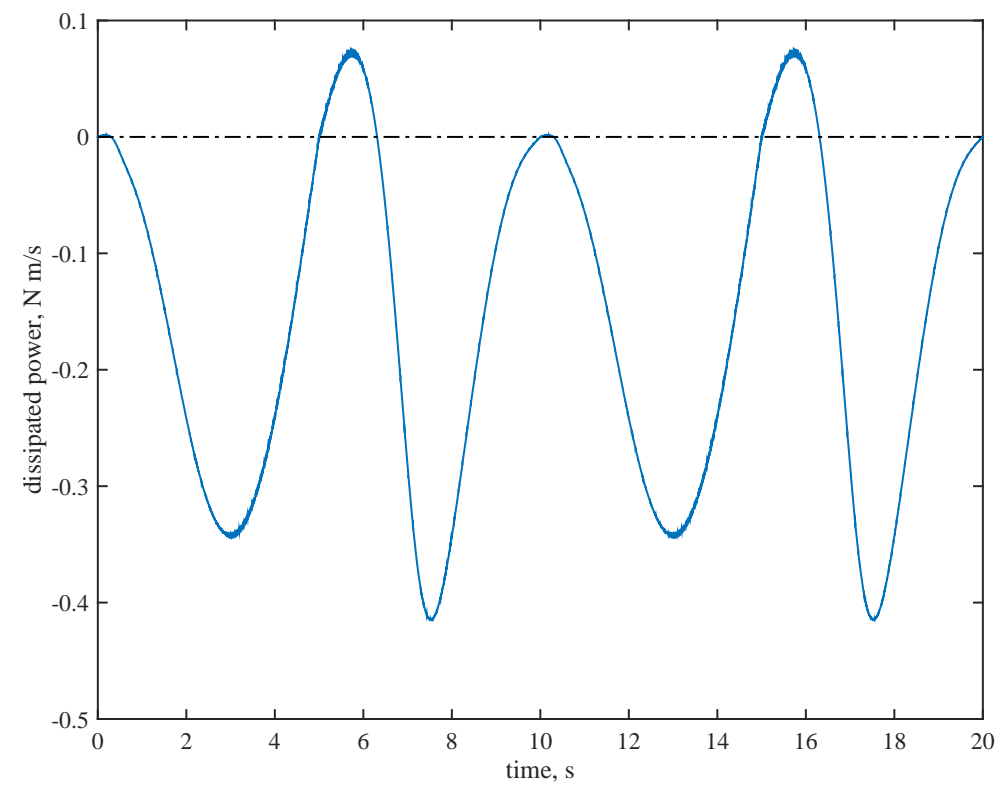

Figure 7: Power for two loading cycles.

LuGre model, an elastic behavior to the micro-asperities, which release elastic energy when the sliding velocity becomes zero.

If $\mu_{s}=\mu_{k}$, i.e. the friction coefficient is constant, the LuGre model adopted turns in the Dahl model that is rate independent as mentioned above. Figure 8 exhibits the dissipative loops for a concrete mixture with Dahl model and frequencies $0.1 \mathrm{~Hz}$ and $1 \mathrm{~Hz}$, and it is easily observable that the area of a dissipative loop does not depend on the frequency.

\section{Conclusions}

In this paper, an already tested mathematical model is applied for describing some dissipation phenomena which are observed in some experimental tests carried out on cylindrical concrete specimens subjected to uniaxial compressive loading. In order to achieve a better match between the model proposed and the experimental tests performed with different frequencies, we propose to exploit a LuGre-type model adapted to a continuum medium to take into account of the observed 'Stribeck effect'. Specifically, for the sake of simplicity, we use a reduced model constituted of three coupled ODEs which respectively describe macroscopic, mesoscopic and microscopic equilibrium of the material modeled.

The numerical simulations that have been carried out show a good agreement with the mea- 

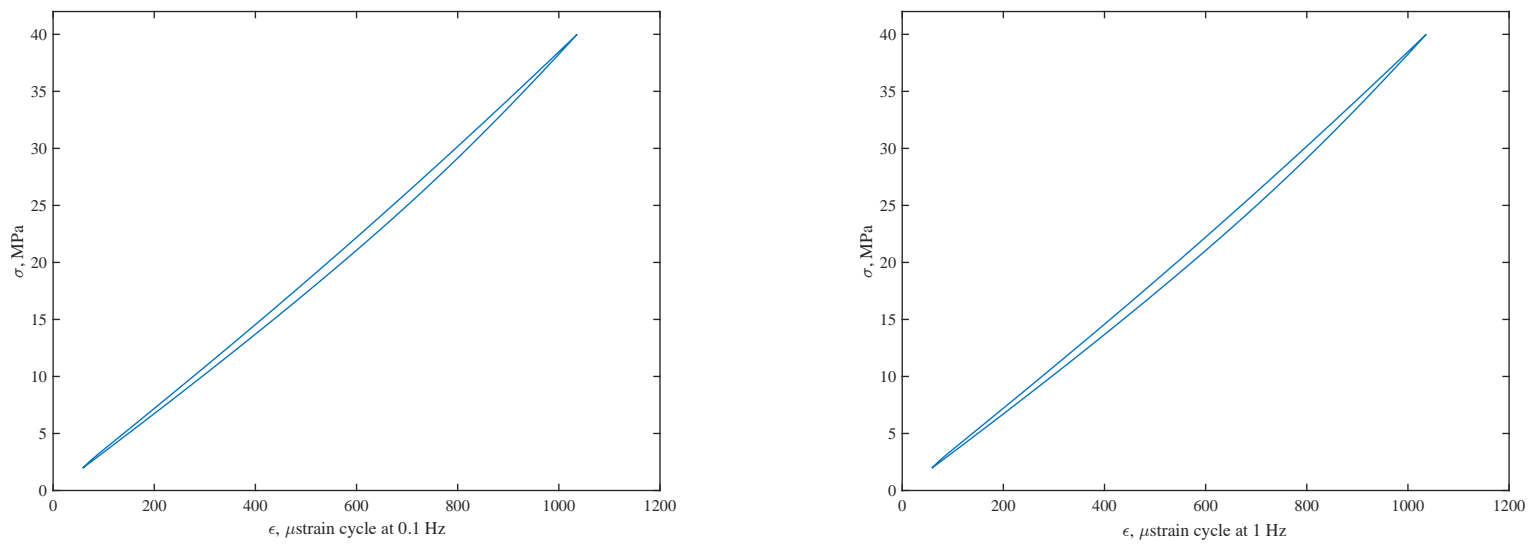

Figure 8: Dissipative loops for a concrete mixture with Dahl model and frequencies $0.1 \mathrm{~Hz}$ and 1 $\mathrm{Hz}$.

surements carried out at different frequencies, allowing us to validate the model proposed in the frequency range explored.

Finally, we have shown that if we consider the Dahl model to describe the friction force instead of the LuGre one, the dissipative loops do not depend on the frequency of the applied load. Thus, with a little change in the model, we can capture different behaviors that can arise from different mixtures or even from other materials that present a micro-structure similar to that considered here.

The model seems to be extendable to interpret friction phenomena in concrete also in conjunction with steel rebars. In this sense, an application to simplified models of concrete beams like the one described in [17] appears to be very interesting and immediately deducible from the model. Thus, further developments are expected considering composite fiber-reinforced models and the interaction between the steel bars and the concrete based on suitable homogenizations (see e.g. [1, 50, 34, 16, $27,42])$.

Acknowledgments The authors would like to thank Félix Darve for helpful comments and advice through the study.

\section{References}

[1] J.-J. Alibert and A. Della Corte. Second-gradient continua as homogenized limit of pantographic microstructured plates: a rigorous proof. Z. Angew. Math. Phys. - ZAMP, DOI 10.1007/s00033015-0526-x, 2015. 
[2] H. Altenbach and V. A. Eremeyev. Analysis of the viscoelastic behavior of plates made of functionally graded materials. ZAMM-Journal of Applied Mathematics and Mechanics/Zeitschrift für Angewandte Mathematik und Mechanik, 88(5):332-341, 2008.

[3] U. Andreaus, P. Baragatti, and L. Placidi. Experimental and numerical investigations of the responses of a cantilever beam possibly contacting a deformable and dissipative obstacle under harmonic excitation. International Journal of Non-Linear Mechanics, doi: 10.1016/j.ijnonlinmec.2015.10.007, 2015.

[4] U. Andreaus, B. Chiaia, and L. Placidi. Soft-impact dynamics of deformable bodies. Continuum Mechanics and Thermodynamics, 25(2-4):375-398, 2013.

[5] N. Barahanov and R. Ortega. Necessary and sufficient conditions for passivity of the LuGre friction model. Automatic Control, IEEE Transactions on, 45(4):830-832, 2000.

[6] A. Berezovski, I. Giorgio, and A. Della Corte. Interfaces in micromorphic materials: Wave transmission and reflection with numerical simulations. Mathematics and Mechanics of Solids, doi: 10.1177/1081286515572244, 2015.

[7] M. A. Biot. Generalized theory of acoustic propagation in porous dissipative media. The Journal of the Acoustical Society of America, 34(9A):1254-1264, 1962.

[8] S. Biswas and A. Chatterjee. A reduced-order model from high-dimensional frictional hysteresis. Proceedings of the Royal Society of London A: Mathematical, Physical and Engineering Sciences, 470(2166):20130817, 2014.

[9] L. C. Bo and D. Pavelescu. The friction-speed relation and its influence on the critical velocity of stick-slip motion. Wear, 82(3):277-289, 1982.

[10] A. Carcaterra and A. Akay. Dissipation in a finite-size bath. Physical Review E, 84(1):011121, 2011.

[11] A. Carcaterra, F. dell'Isola, R. Esposito, and M. Pulvirenti. Macroscopic description of microscopically strongly inhomogenous systems: A mathematical basis for the synthesis of higher gradients metamaterials. Archive for Rational Mechanics and Analysis, 218(3):1239-1262, 2015.

[12] A. Carcaterra, N. Roveri, and G. Pepe. Fractional dissipation generated by hidden wave-fields. Mathematics and Mechanics of Solids, doi: 10.1177/1081286513518941, 2014.

[13] A. Cazzani, M. Malagù, and E. Turco. Isogeometric analysis: a powerful numerical tool for the elastic analysis of historical masonry arches. Continuum Mechanics and Thermodynamics, pages 1-18, 2014.

[14] A. Cazzani, M. Malagù, E. Turco, and F. Stochino. Constitutive models for strongly curved beams in the frame of isogeometric analysis. Mathematics and Mechanics of Solids, doi: $10.1177 / 1081286515577043,2015$. 
[15] A. Cazzani and P. Ruge. Numerical aspects of coupling strongly frequency-dependent soilfoundation models with structural finite elements in the time-domain. Soil Dynamics and Earthquake Engineering, 37:56-72, 2012.

[16] AF Cheviakov, J-F Ganghoffer, and S St Jean. Fully non-linear wave models in fiber-reinforced anisotropic incompressible hyperelastic solids. International Journal of Non-Linear Mechanics, $71: 8-21,2015$.

[17] L. Contrafatto, M. Cuomo, and F. Fazio. An enriched finite element for crack opening and rebar slip in reinforced concrete members. International journal of fracture, 178(1-2):33-50, 2012.

[18] S. C. Cowin and J. W. Nunziato. Linear elastic materials with voids. Journal of Elasticity, 13(2):125-147, 1983.

[19] M. Cuomo, L. Contrafatto, and L. Greco. A variational model based on isogeometric interpolation for the analysis of cracked bodies. International Journal of Engineering Science, 80:173-188, 2014.

[20] P. R. Dahl. A solid friction model. Technical Report No. TOR-0158 (3107-18)-1, DTIC Document. AEROSPACE CORP EL SEGUNDO CA, 1968.

[21] P. R. Dahl. Solid friction damping of mechanical vibrations. AIAA Journal, 14(12):1675-1682, 1976.

[22] H. Dankowicz. On the modeling of dynamic friction phenomena. ZAMM-Journal of Applied Mathematics and Mechanics/Zeitschrift für Angewandte Mathematik und Mechanik, 79(6):399409, 1999.

[23] C. C. De Wit, H. Olsson, K. J. Astrom, and P. Lischinsky. A new model for control of systems with friction. Automatic Control, IEEE Transactions on, 40(3):419-425, 1995.

[24] D. Del Vescovo and A. Fregolent. Theoretical and experimental dynamic analysis aimed at the improvement of an acoustic method for fresco detachment diagnosis. Mechanical Systems and Signal Processing, 23(7):2312-2319, 2009.

[25] A. Della Corte, A. Battista, and F. dell'Isola. Referential description of the evolution of a 2D swarm of robots interacting with the closer neighbors: perspectives of continuum modeling via higher gradient continua. International Journal of Non-Linear Mechanics, (doi: 10.1016/j.ijnonlinmec.2015.06.016), 2015.

[26] F. dell'Isola and R. C. Batra. Saint-Venant's problem for porous linear elastic materials. Journal of elasticity, 47(1):73-81, 1997.

[27] F. dell'Isola, A. Della Corte, L. Greco, and A. Luongo. Plane bias extension test for a continuum with two inextensible families of fibers: A variational treatment with Lagrange 
multipliers and a perturbation solution. International Journal of Solids and Structures, doi: 10.1016/j.ijsolstr.2015.08.029, 2015.

[28] F. dell'Isola and K. Hutter. What are the dominant thermomechanical processes in the basal sediment layer of large ice sheets? Proceedings of the Royal Society of London. Series A: Mathematical, Physical and Engineering Sciences, 454(1972):1169-1195, 1998.

[29] F. dell'Isola, A. Madeo, and P. Seppecher. Cauchy tetrahedron argument applied to higher contact interactions. doi: 10.1007/s00205-015-0922-6, 2015.

[30] F. dell'Isola and A. Romano. A phenomenological approach to phase transition in classical field theory. International journal of engineering science, 25(11):1469-1475, 1987.

[31] F. dell'Isola, L. Rosa, and C. Woźniak. Dynamics of solids with microperiodic nonconnected fluid inclusions. Archive of Applied Mechanics, page 14, 1997.

[32] F. dell'Isola, L. Rosa, and C. Woźniak. A micro-structured continuum modelling compacting fluid-saturated grounds: The effects of pore-size scale parameter. Acta mechanica, 127(1-4):165182, 1998.

[33] F. dell'Isola, P. Seppecher, and A. Della Corte. The postulations á la D'Alembert and á la Cauchy for higher gradient continuum theories are equivalent: a review of existing results. Proceedings of the Royal Society of London A, 471(2183):20150415, 2015.

[34] F. dell'Isola and D. Steigmann. A two-dimensional gradient-elasticity theory for woven fabrics. Journal of Elasticity, 118(1):113-125, 2015.

[35] L. Dietrich, T. Lekszycki, and K. Turski. Problems of identification of mechanical characteristics of viscoelastic composites. Acta mechanica, 126(1-4):153-167, 1998.

[36] U. El Shamy and C. Denissen. Microscale energy dissipation mechanisms in cyclically-loaded granular soils. Geotechnical and Geological Engineering, 30(2):343-361, 2012.

[37] V. A. Eremeyev. On effective properties of materials at the nano-and microscales considering surface effects. Acta Mechanica, DOI: 10.1007/s00707-015-1427-y, 2015.

[38] S. Federico, A. Grillo, and S. Imatani. The linear elasticity tensor of incompressible materials. Mathematics and Mechanics of Solids, 20(6):643-662, 2015.

[39] S. Federico, A. Grillo, S. Imatani, G. Giaquinta, and W. Herzog. An energetic approach to the analysis of anisotropic hyperelastic materials. International Journal of Engineering Science, 46(2):164-181, 2008.

[40] S. Federico, A. Grillo, and G. Wittum. Considerations on incompressibility in linear elasticity. Il Nuovo cimento della Societa italiana di fisica. C, 32(1):81, 2009. 
[41] L. Greco and M. Cuomo. B-Spline interpolation of Kirchhoff-Love space rods. Computer Methods in Applied Mechanics and Engineering, 256:251-269, 2013.

[42] Z. Guo, X. Shi, Y. Chen, H. Chen, X. Peng, and P. Harrison. Mechanical modeling of incompressible particle-reinforced neo-Hookean composites based on numerical homogenization. Mechanics of Materials, 70:1-17, 2014.

[43] F. Hernandez-Olivares, G. Barluenga, M. Bollati, and B. Witoszek. Static and dynamic behaviour of recycled tyre rubber-filled concrete. Cement and concrete research, 32(10):1587-1596, 2002 .

[44] B. Jacobson. The Stribeck memorial lecture. Tribology International, 36(11):781-789, 2003.

[45] G. Jouan, P. Kotronis, and F. Collin. Using a second gradient model to simulate the behaviour of concrete structural elements. Finite Elements in Analysis and Design, 90:50-60, 2014.

[46] M. L. Kachanov. A microcrack model of rock inelasticity part I: Frictional sliding on microcracks. Mechanics of Materials, 1(1):19-27, 1982.

[47] C.-H. Lamarque, F. Bernardin, and J. Bastien. Study of a rheological model with a friction term and a cubic term: deterministic and stochastic cases. European Journal of Mechanics-A/Solids, 24(4):572-592, 2005.

[48] A. Luongo and G. Piccardo. A continuous approach to the aeroelastic stability of suspended cables in 1:2 internal resonance. Journal of Vibration and Control, 14(1-2):135-157, 2008.

[49] A. Madeo. Effect of micro-particle additions on frictional energy dissipation and strength of concrete. Master thesis, 2006.

[50] A. Madeo, A. Della Corte, L. Greco, and P. Neff. Wave propagation in pantographic 2D lattices with internal discontinuities. Proceedings of the Estonian Academy of Sciences, 64(3S):325-330, 2015 .

[51] A. Madeo, T. Lekszycki, and F. dell'Isola. A continuum model for the bio-mechanical interactions between living tissue and bio-resorbable graft after bone reconstructive surgery. Comptes Rendus Mécanique, 339(10):625-640, 2011.

[52] C. Makkar, W. E. Dixon, W. G. Sawyer, and G. Hu. A new continuously differentiable friction model for control systems design. In Advanced Intelligent Mechatronics. Proceedings, 2005 IEEE/ASME International Conference on, pages 600-605. IEEE, 2005.

[53] C. Maurini, J. Pouget, and F. del'Isola. Extension of the Euler-Bernoulli model of piezoelectric laminates to include 3D effects via a mixed approach. Computers \& structures, 84(22):14381458, 2006. 
[54] P. K. Mehta and P. J. M. Monteiro. Concrete: microstructure, properties, and materials, volume 3. McGraw-Hill New York, 2006.

[55] A. Misra. Stabilization characteristics of clays using class C fly ash. Transportation Research Record: Journal of the Transportation Research Board, 1611(1):46-54, 1998.

[56] G. Oliveto and M. Cuomo. Incremental analysis of plane frames with geometric and material nonlinearities. Engineering Structures, 10(1):2-12, 1988.

[57] H. Olsson, K. J. Åström, C. C. De Wit, M. Gäfvert, and P. Lischinsky. Friction models and friction compensation. European journal of control, 4(3):176-195, 1998.

[58] A. K. Padthe, J. Oh, and D. S. Bernstein. On the LuGre model and friction-induced hysteresis. In Proceedings of the 2006 American Control Conference Minneapolis, Minnesota, USA, 2006.

[59] V. Pensée, D. Kondo, and L. Dormieux. Micromechanical analysis of anisotropic damage in brittle materials. Journal of Engineering Mechanics, 128(8):889-897, 2002.

[60] G. Piccardo and G. Solari. A refined model for calculating 3-D equivalent static wind forces on structures. Journal of wind engineering and industrial aerodynamics, 65(1):21-30, 1996.

[61] L. Placidi. A variational approach for a nonlinear one-dimensional damage-elasto-plastic secondgradient continuum model. Continuum Mechanics and Thermodynamics, (doi:10.1007/s00161014-0405-2), 2014.

[62] L. Placidi. A variational approach for a nonlinear 1-dimensional second gradient continuum damage model. Continuum Mechanics and Thermodynamics, 27(4):623-638, 2015.

[63] L. Placidi, U. Andreaus, A. Della Corte, and T. Lekszycki. Gedanken experiments for the determination of two-dimensional linear second gradient elasticity coefficients. Zeitschrift für angewandte Mathematik und Physik, 66(6):3699-3725, 2015.

[64] L. Placidi, F. dell'Isola, N. Ianiro, and G. Sciarra. Variational formulation of pre-stressed solidfluid mixture theory, with an application to wave phenomena. European Journal of MechanicsA/Solids, 27(4):582-606, 2008.

[65] L. Placidi, S. H. Faria, and K. Hutter. On the role of grain growth, recrystallization and polygonization in a continuum theory for anisotropic ice sheets. Annals of Glaciology, 39(1):49$52,2004$.

[66] L. Placidi and K. Hutter. Thermodynamics of polycrystalline materials treated by the theory of mixtures with continuous diversity. Continuum Mechanics and Thermodynamics, 17(6):409451, 2006. 
[67] M. Porfiri, F. dell'Isola, and E. Santini. Modeling and design of passive electric networks interconnecting piezoelectric transducers for distributed vibration control. International Journal of Applied Electromagnetics and Mechanics, 21(2):69-87, 2005.

[68] N. L. Rizzi and V. Varano. On the postbuckling analysis of thin-walled frames. 2011.

[69] N.L. Rizzi, V. Varano, and S. Gabriele. Initial postbuckling behavior of thin-walled frames under mode interaction. Thin-Walled Structures, 68:124-134, 2013.

[70] G. C. Ruta, V. Varano, M. Pignataro, and N. L. Rizzi. A beam model for the flexural-torsional buckling of thin-walled members with some applications. Thin Wall. Struct., 46(7-9):816-822, 2008 .

[71] D. Scerrato. Effect of Micro-Particle Addition on Frictional Energy Dissipation and Strength of Concrete: Experiments and Modelling. Phd thesis, Theoretical and Applied Mechanics and Mécanique, Énergétique, Génie Civil, Acoustique, 2014.

[72] D. Scerrato, I. Giorgio, A. Della Corte, A. Madeo, and A. Limam. A micro-structural model for dissipation phenomena in the concrete. International Journal for Numerical and Analytical Methods in Geomechanics, 39(18):2037-2052, 2015.

[73] D. Scerrato, I. Giorgio, A. Madeo, A. Limam, and F. Darve. A simple non-linear model for internal friction in modified concrete. International Journal of Engineering Science, 80:136-152, 2014 .

[74] G. Sciarra, F. dell'Isola, and K. Hutter. A solid-fluid mixture model allowing for solid dilatation under external pressure. Continuum Mechanics and Thermodynamics, 13(5):287-306, 2001.

[75] A. Signorini. Questioni di elasticità non linearizzata e semilinearizzata. Rend. Mat. Appl., V. Ser., 18:95-139, 1959.

[76] R. Stribeck and M. Schröter. Die wesentlichen Eigenschaften der Gleit-und Rollenlager: Untersuchung einer Tandem-Verbundmaschine von 1000 PS. Springer, 1903.

[77] J. Swevers, F. Al-Bender, C. G. Ganseman, and T. Projogo. An integrated friction model structure with improved presliding behavior for accurate friction compensation. Automatic Control, IEEE Transactions on, 45(4):675-686, 2000.

[78] D. Tonazzi, F. Massi, L. Baillet, A. Culla, M. Di Bartolomeo, and Y. Berthier. Experimental and numerical analysis of frictional contact scenarios: from macro stick-slip to continuous sliding. Meccanica, 50:649-664, 2015.

[79] E. Turco and M. Aristodemo. A three-dimensional B-spline boundary element. Computer methods in applied mechanics and engineering, 155(1):119-128, 1998. 
[80] J. Wojewoda, A. Stefański, M. Wiercigroch, and T. Kapitaniak. Hysteretic effects of dry friction: modelling and experimental studies. Philosophical Transactions of the Royal Society A: Mathematical, Physical and Engineering Sciences, 366(1866):747-765, 2008.

[81] A. Wrzesniak, C. Dascalu, and P. Bésuelle. A two-scale time-dependent model of damage: Influence of micro-cracks friction. European Journal of Mechanics-A/Solids, 49:345-361, 2015.

[82] Y. Yang and A. Misra. Micromechanics based second gradient continuum theory for shear band modeling in cohesive granular materials following damage elasticity. International Journal of Solids and Structures, 49(18):2500-2514, 2012.

[83] V. A. Yeremeyev, A. B. Freidin, and L. L. Sharipova. The stability of the equilibrium of two-phase elastic solids. Journal of applied mathematics and mechanics, 71(1):61-84, 2007.

[84] Q. Zhu, D. Kondo, J. Shao, and V. Pensée. Micromechanical modelling of anisotropic damage in brittle rocks and application. International Journal of Rock Mechanics and Mining Sciences, 45(4):467-477, 2008. 